\title{
Regulation of heat shock protein 70-1 expression by androgen receptor and its signaling in human prostate cancer cells
}

\author{
SHAN LU ${ }^{1}$, ZONGQIN TAN ${ }^{1}$, MATT WORTMAN $^{2}$, SHAN LU $^{3}$ and ZHONGYUN DONG ${ }^{1}$ \\ Departments of ${ }^{1}$ Internal Medicine, ${ }^{2}$ Cancer and Cell Biology, and ${ }^{3}$ Pathology and Laboratory Medicine, \\ The University of Cincinnati College of Medicine, Cincinnati, OH 45267, USA
}

Received October 8, 2009; Accepted November 23, 2009

DOI: 10.3892/ijo_00000520

\begin{abstract}
Heat shock protein (hsp) 70-1 (hsp70-1) is overexpressed in human prostate cancer cells and may play important roles in prostate cancer resistance to conventional therapies. The purpose of this study was to investigate whether androgen receptor (AR) and its signaling regulate hsp70-1 expression. Several lines of AR-positive (LNCaP, LAPC-4, and 22Rv1) and -negative (PC-3, DU145, WPE1-NB14 and WPE1-NB-26) human prostatic cells were used in the study. Dihydrotestosterone (DHT) enhanced hsp70-1 expression in LNCaP cells. Expression of hsp70-1 in LNCaP cells was downregulated by the anti-androgens bicalutamide (Bic), and flutamide (Flut), and a newly identified AR signaling antagonist DL3. The downregulation of hsp70-1 by DL3 was also observed in LAPC-4 and 22Rv1 cells, but not in the four lines of AR-negative cells examined. Expression of hsp70-1 was also reduced by DL3 in PC-3 cells engineered with AR. On the other hand, knocking down AR in LNCaP cells by siRNA moderately reduced hsp70-1 level and abolished effects of DL3 on hsp70-1 expression. DL3 reduced hsp70-1 mRNA synthesis in cells and its in vitro gene transcription but did not significantly alter the stabilities of hsp70-1 mRNA and protein. Chromatin-immunoprecipitation (ChIP) assay showed that AR bound to the promoter region of $H S P A 1 B$ gene, which was reduced in cells treated with DL3 or Bic. These data suggest that AR and its signaling regulate hsp70-1
\end{abstract}

Correspondence to: Dr Zhongyun Dong, Department of Internal Medicine, The University of Cincinnati College of Medicine, 3125 Eden Ave., Rm. 1422, Cincinnati, OH 45267, USA

E-mail: dongzu@ucmail.uc.edu

Abbreviations: AR, androgen receptor; Bic, bicalutamide; ChIP, chromatin immunoprecipitation; DHT, dihydrotestosterone; DL3, 6-amino-2-[2-(4-tert-butyl-pnenoxy)-ethylsulfanyl]-1H-pyrimidin4-one; ELISA, enzyme-linked immunoabsorbance assay; FBS, fetal bovine serum; Flut, flutamide; hsp, heat shock protein; HSF1, heat shock factor 1; PSA, prostate-specific antigen; qRT-PCR, quantitative real-time reverse-transcription PCR

Key words: androgen receptor, heat shock protein 70-1, antagonist, prostate cancer expression in prostate cancer cells and that $H S P A 1 B$ could be an AR target gene.

\section{Introduction}

Heat shock proteins (hsp) are encoded by several gene families and play essential roles in cell survival, tumorigenesis, and tumor progression $(1,2)$. The hsp70 family proteins, named according to their approximate relative molecular mass, contain at least 8 members that are almost ubiquitously expressed $(3,4)$. The two major hsp70 proteins are hsp70-1a and hsp70-1b with amino acids being $>99 \%$ identical and are encoded by HSPAIA and HSPAIB genes, respectively $(3,4)$. They were initially found in cells under stress $(3,4)$. There are also several hsp70 homologues that are constitutively expressed and are termed as heat shock 'cognate' proteins (Hscs). Hsc70 and hsp70 are highly homologous and have identical biochemical properties (5) and similar domain structures, including an $\mathrm{N}$-terminal ATPase domain, a peptide-binding domain with the EEVD motif mediating hsp70 interaction with tetratricopeptide repeat (TPR)-containing client protein, and a C-terminus (5). Along with hsp90 and several co-chaperones, hsp70 plays critical roles in maintaining protein integrity and synthesis, such as folding and assembling AR to a high-affinity ligand binding conformation $(1,6-8)$.

Hsp70-1 proteins are overexpressed in many types of tumor and this overexpression of hsp70-1 is often correlated with tumor malignancy, and progression $(1,5,9)$, poor prognosis and presence of metastasis $(1,5,9,10)$. Overexpression of hsp70-1 induces cell transformation $(5,11,12)$. These effects of hsp70-1, possibly through downregulation of mammalian sterile 20-like kinase 1 (13), are due partially to its protection of cells from apoptosis and/or necrosis induced by chemotherapeutic agents and other stress stimuli (14-16). Indeed, depletion of hsp70-1 expression was shown to be sufficient to trigger apoptosis in several types of cells (17). Hsp70-1 expression is elevated in prostate cancer (9). Although depletion of hsp70-1 using siRNA and antisense RNA did not alter the cell viability, it did enhance sensitivity of prostate cancer cells to a variety of anticancer agents (9), suggesting that downregulation of hsp70-1 could be an effective approach for combination therapies to overcome the natural resistance of prostate cancer to chemotherapies.

Expression of hsp70-1 is mainly regulated by heat shock factor 1 (HSF1) that binds to heat shock transcription control 
element. Interestingly, it has been shown that androgen, through activation of AR, induces hsp70-1 expression in ventricular myocytes (18). Moreover, overexpression of AR and other steroid receptors has been shown to activate HSF1 in COS-1 fibroblasts (19). These observations clearly suggest that AR signaling regulates hsp70-1 expression.

The purpose of this study was to determine potential roles of AR signaling in regulation of hsp70-1 expression in prostate cancer cells. We show that DHT enhanced hsp70-1 mRNA expression, which was attenuated by a newly identified AR signaling antagonist DL3 (20). Expression of hsp70-1 protein was also downregulated by DL3 and the non-steroid antiandrogens Bic and Flut. DL3-induced downregulation of hsp70-1 was more moderate in AR-negative cells and was enhanced when AR-negative cells are forced to overexpress AR. Treatment with DL3 reduced hsp70-1 mRNA gene transcription but had marginal effects on hsp70-1 mRNA stability. Finally, AR bound to promoter region of the HSPAlB gene, which was attenuated by DL3 and, to a lower extent, by Bic.

\section{Materials and methods}

Tumor cells and culture. LNCaP (21,22), 22Rv1 (22,23), WPE1-NB14 and WPE1-NB26, PC-3, and DU-145 cells were purchased from American Type Culture Collection (ATCC, Manassas, VA). LNCaP and 22Rv1 cells were maintained in RPMI-1640 supplemented with $10 \%$ FBS. WPE1-NB14 and WPE1-NB-26 cells were cultured in the keratinocyte serum-free medium (K-SFM) supplemented with bovine pituitary extract $(50 \mu \mathrm{g} / \mathrm{ml})$ and epidermal growth factor $(5 \mathrm{ng} / \mathrm{ml})$. PC-3 and DU-145 cells were cultured in MEM supplemented with 5\% FBS. PC-3AR cells, derived from PC-3 cells by stable transfection of wild-type AR, were generously provided by Dr Zhengxin Wang (Department of Cancer Biology, The University of Texas M.D. Anderson Cancer Center, Houston, TX). LAPC-4 cells $(22,24)$ were generously provided by Dr Karen Knudsen (Kimmel Cancer Center, Thomas Jefferson Medical College, Philadelphia, PA). LAPC-4 cells were maintained in IMDM medium supplemented with $10 \%$ FBS and $10 \mathrm{nM}$ of DHT.

Immunoblot analysis. Cells (2×10\%/60-mm dish) were plated and treated after an overnight incubation as desired. The cells were washed in cold PBS/5 mM EDTA, scraped into a lysis buffer, and analyzed by Western blotting (25) using antibodies against PSA (DakoCytomation, Glostrup, Denmark), AR (Epitomics, Burlingame, CA), hsp70-1 (Cell Signaling Technology, Inc., Danvers, MA), hsp90 (Santa Cruz Biotechnology, Santa Cruz, CA), and B-actin (Santa Cruz). The immunoreactive signals were revealed using an ECL Western blotting detection system (Michigan Diagnostic LLC, Troy, MI) and visualized in a Kodak IS4000MM Image Station (Eastman Kodak Co., Rochester, NY).

qRT-PCR. Cells were seeded at $2 \times 10^{6}$ cells $/ 60-\mathrm{mm}$ dish. After an overnight incubation, the cells were treated in fresh medium for various times, washed, and total RNA was extracted using TRIzol reagent. Expressions of AR, PSA, and hsp70-1 were analyzed by qRT-PCR (26) using the following primer pairs: AR, 5'-GGTGAGCAGAGTGCCCT ATC-3'/5'-GGCAGTCTCCAAACGCATGTC-3'; PSA, 5'GACCACCTGCTACGCCTCA-3'/5'-AACTTGCGCACAC ACGTCATT-3'; hsp70-1, 5'-CGAGGCGGACAAGAA GAA-3'/5'-GATGGGGTTACACACCTGCTC-3' and $ß$-actin, 5'-CGGCACCACCATGTACCCT-3'/5'-ACACGGAGTA CTTGCGCTCAG-3', respectively. The cycle threshold values were used to calculate the normalized gene expression against B-actin using the Q-Gene software.

Nuclear run-on assay. A qRT-PCR-based assay was performed (26). Briefly, cells were rinsed with cold PBS and scraped in lysis buffer on ice, followed by addition of the same volume of $0.6 \mathrm{M}$ sucrose buffer. Cell nucleus pellets, obtained by centrifugation at $500 \mathrm{x}$ g for $10 \mathrm{~min}$, were incubated for $30 \mathrm{~min}$ at $30^{\circ} \mathrm{C}$ in $500 \mu \mathrm{l}$ of transcription reaction buffer $(\mathrm{pH} 7.9)$ containing $50 \mathrm{mM}$ Tris, $5 \mathrm{mM} \mathrm{MgCl}_{2}, 0.1 \mathrm{mM}$ EDTA, and $0.5 \mathrm{mM}$ NTP. RNA was extracted and followed by DNase treatment and qRT-PCR. Samples without transcription reaction were used as controls.

Transient transfection. Cells (10\%60-mm dish) were transiently transfected with an expression vector encoding human hsp70-1 (pCMV6-XL5, OriGene, Rockville, MD), AR-specific siRNA (On-Target plus Smartpool), or a scrambled control siRNA (Dharmacon, Inc., Chicago, IL) using the Lipofectamine 2000 transfection reagent (Invitrogen, Carlsbad, CA). Twentyfour to $48 \mathrm{~h}$ later, expressions of $\mathrm{AR}$ and hsp70-1 were determined by immunoblotting.

Chromatin immunoprecipitation (ChIP) assay. Cells were plated into $150 \mathrm{~mm}$ dish $\left(1.5 \times 10^{7} /\right.$ plate $)$. After an overnight incubation, the cells were treated for $4 \mathrm{~h}$ with $20 \mu \mathrm{M}$ of DL3 or Bic, fixed in $1 \%$ formaldehyde for $10 \mathrm{~min}$ at room temperature, rinsed with cold PBS, and incubated in PBS containing $125 \mathrm{mM}$ glycine for $5 \mathrm{~min}$. The cells were then scrapped into PBS supplemented with $5 \mathrm{mM}$ PMSF and pelleted. The ChIP assay was carried out using a kit from Upstate Biotechnology (Lake Placid, USA). Briefly, cells were lysed in SDS lysis buffer (1\% SDS, 10 mM EDTA, $50 \mathrm{mM}$ Tris- $\mathrm{HCl}, \mathrm{pH} 8.1$ ) with protease inhibitors and sonicated using Sonicator S-4000 (Misonix, NY) to sheer DNA. AR-chromatin-transcription factor complex was precipitated with an antibody to AR (C19, Santa Cruz) or normal rabbit IgG (control). With 1:150 dilution of total DNA as a positive control (total input), DNA obtained from the immunoprecipitation was analyzed by using qPCR. The primer pair encoding the promoter region of human $H S P A 1 B$ gene (5'-GGTCCGCTTCGTCTTTCG-3'/5'-CTCTGTGGG CTC CGCTCT-3') was published by others (27) (accession no. NM005346). Data from ChIP assay were presented as the ratio between precipitated DNA over total input. A portion of the ChIP samples was analyzed by immunoblotting to evaluate AR levels. In a parallel setting, total cell lysates were precipitated with the same AR antibody to evaluate the total cellular AR level in each treatment group.

Statistical analysis. All experiments were performed at least twice. Data shown are the mean \pm SE. Differences between means were compared using the two-tailed Student's t-test 
A

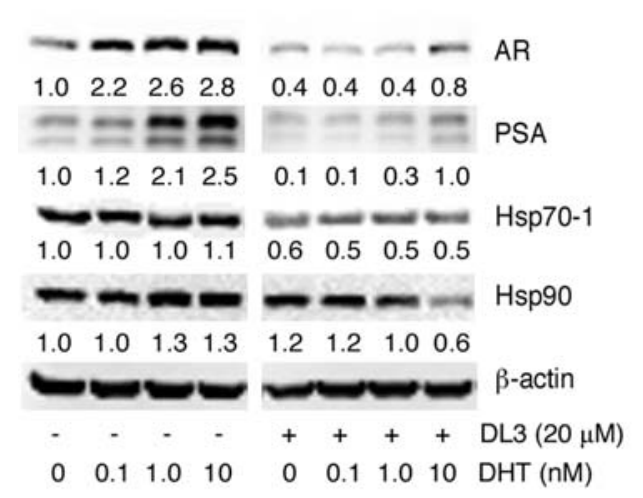

B

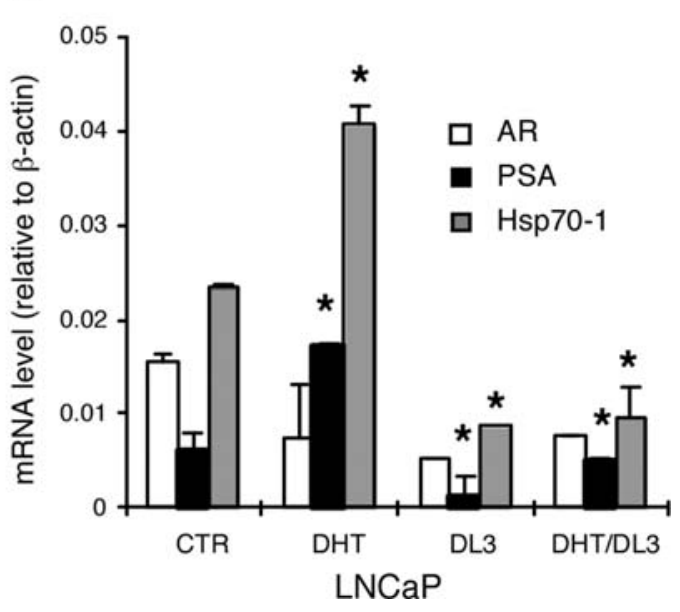

Figure 1. Effects of DHT and DL3 on hsp70 and hsp90 expression. (A) LNCaP cells were incubated for 48 h in phenol-free medium supplemented with $10 \%$ charcoal-dextran stripped FBS (SFBS), then treated for $24 \mathrm{~h}$ with $0-10 \mathrm{nM}$ of DHT in the absence or presence of $20 \mu \mathrm{M}$ of DL3. Cell lysates were analyzed by immunoblotting with antibodies to AR, PSA, hsp70-1 and hsp90 with B-actin as a loading control. (B) LNCaP cells were treated as described in (A). Total RNA was extracted and expression of AR, PSA, hsp70-1 with B-actin as a loading control was determined by qRT-PCR and presented as ratios to ß-actin. " $\mathrm{p}<0.05$ : DHT vs. CTR; DL3 vs. CTR; DHT/DL3 vs. DHT. Data shown were one representative experiment of three.

and were considered significantly different at the level of $\mathrm{p}<0.05$.

\section{Results}

Effects of DHT and DL3 on AR, PSA, and hsp70-1 expression. Expression of heat shock proteins, including hsp70-1 and hsp90, are elevated in human prostate cancer cells. Regulation of their expression is, however, not well studied. We determined whether hsp70-1 and hsp90 expression in LNCaP cells was regulated by AR signaling in cells treated with DHT and DL3, a novel AR signaling antagonist identified in our previous study (20). LNCaP cells were incubated for $48 \mathrm{~h}$ in medium supplemented with $10 \%$ SFBS, then treated for $24 \mathrm{~h}$ in fresh medium supplemented with the SFBS with increasing concentrations of DHT in the absence or presence of $20 \mu \mathrm{M}$ of DL3. The cellular protein was analyzed by immunoblotting. As shown in Fig. 1A, DHT enhanced AR and PSA protein levels in a dose-dependent manner. These effects of DHT were abrogated by the presence of DL3. In contrast to the expression of AR and PSA, expression of neither hsp70-1 nor hsp90 was significantly altered by DHT (Fig. 1A). Treatment with DL3 reduced hsp70-1 expression, regardless of the absence or presence of DHT (Fig. 1A). In contrast, DL3 decreased hsp90 expression only in the presence of $10 \mathrm{nM}$ DHT, a concentration of androgen much higher than those physiologically achievable levels. The qRT-PCR analysis revealed that treatment of DHT (1 nM) did not significantly alter AR mRNA expression but enhanced the expression of both PSA and hsp70-1 mRNA (Fig. 1B). The DHT-enhanced expression of both PSA and hsp70-1 mRNA was attenuated by the presence of DL3 (Fig. 1B).

Downregulation of hsp70-1 expression by DL3 and the antiandrogens. To further explore potential roles of AR signaling in regulation of hsp70-1 and hsp90 expression, the time course and does-dependent effects of DL3 were determined.
LNCaP cells in complete medium were plated and treated with $20 \mu \mathrm{M}$ of DL3. At various times after the treatment, the cellular protein was harvested and analyzed by immunoblotting. As expected, DL3 downregulated expressions of both AR and PSA in a time-dependent manner. Expression of AR and PSA were reduced by 33 and $62 \%$, respectively, at $8 \mathrm{~h}$ after the treatment and further decreased thereafter. Expression of PSA was diminished in cells treated with DL3 for $24 \mathrm{~h}$ (Fig. 2A). These observations confirmed findings reported in our previous study (20). Expression of hsp70-1 was reduced by $\sim 50 \%$. This occurred, however, only in the cells treated by DL3 for 24 and 48 h (Fig. 2A). In contrast, treatment of DL3 did not significantly alter hsp90 expression at any times studied (Fig. 2A). The dose-response analysis showed that expression of PSA was more sensitive to the inhibitory effects of DL3 in comparison with those of AR and hsp70-1. Whereas a significant reduction of PSA was observed in cells treated by $5 \mu \mathrm{M}$ of DL3 (by 70\%), downregulation of both AR and hsp70-1 was observed in cells exposed to DL3 at concentrations $>10 \mu \mathrm{M}$ (Fig. 2B). Consistent with the time course study above, it appeared that DL3-induced downregulation of AR protein expression was relatively more significant than that of hsp70-1 (Fig. 1B). To validate that downregulation of hsp70-1 by DL3 was due to an attenuation of AR signaling, the effects of Bic and Flut, the two non-steroid antiandrogens most commonly used in prostate cancer therapies, on hsp70-1 expression were determined. As shown in Fig. 2C, treatment with both Bic and Flut reduced hsp70-1 expression in LNCaP cells. In agreement with their effects on AR and PSA expression, downregulation of hsp70-1 by these two drugs was relatively more moderate in comparison with that of DL3 (Fig. 2C).

The qRT-PCR analysis of cells cultured for up to $48 \mathrm{~h}$ showed that AR mRNA levels were increased in the first $24 \mathrm{~h}$ after incubation in fresh medium and moderately reduced during the second $24 \mathrm{~h}$ (Fig. 2D). An elevation of both PSA (Fig. 2E) and hsp70-1 (Fig. 2F) mRNA was also observed, 


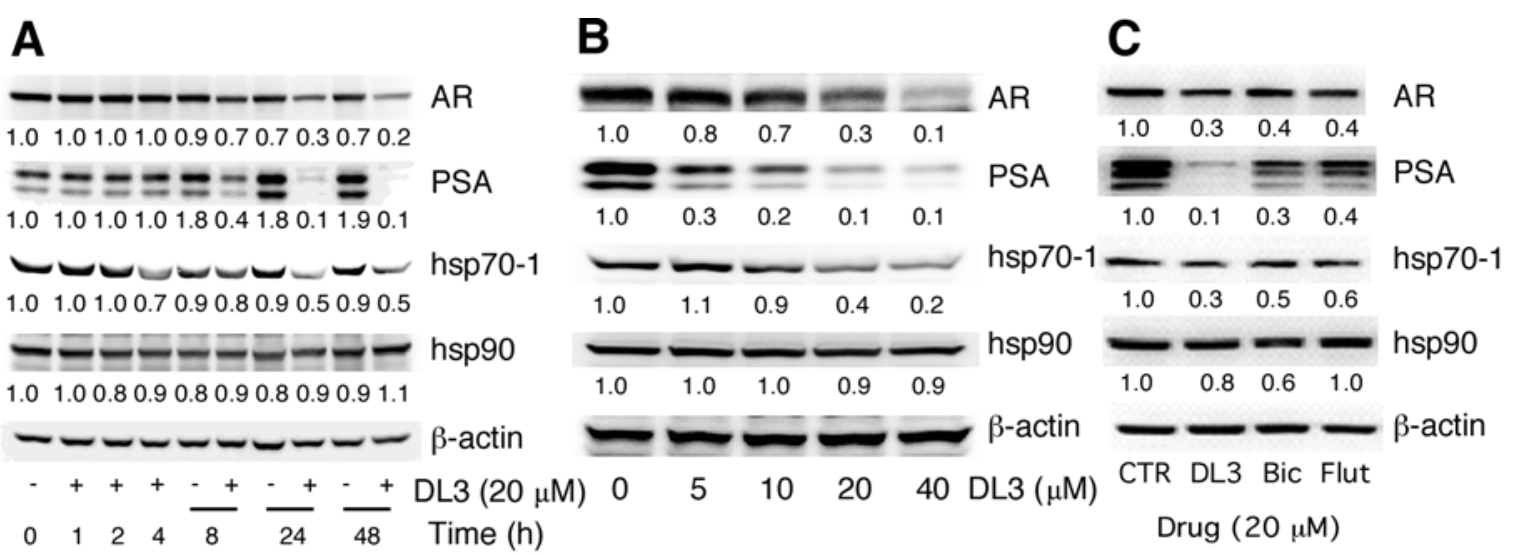

D

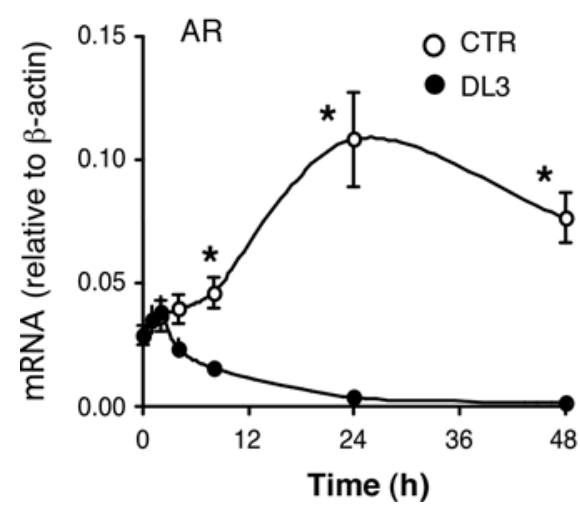

E

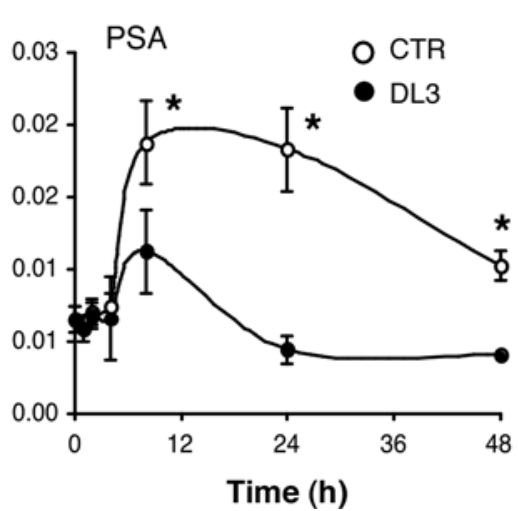

$\mathbf{F}$

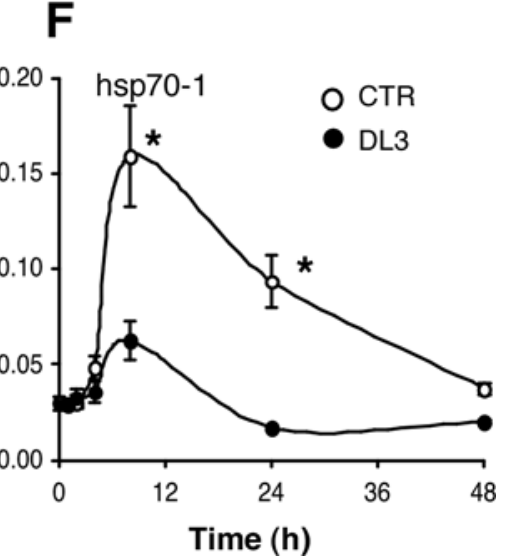

Figure 2. Time- and dose-dependent effects of DL3 on hsp70-1 expression. (A) LNCaP cells were treated for different times in medium supplemented with normal FBS. Cell lysates were analyzed by immunoblotting. (B) Lysates from LNCaP cells treated for $24 \mathrm{~h}$ with different concentrations of DL3 were analyzed by immunoblotting. (C) Lysates from LNCaP cells treated for $24 \mathrm{~h}$ with $20 \mu \mathrm{M}$ of DL3, Bic, or Flut were analyzed by immunoblotting. (D and E) LNCaP cells were incubated for various times in medium supplemented with normal FBS or treated with $20 \mu \mathrm{M}$ of DL3. Total RNA extracted was analyzed by qRT-PCR for the presence of AR (D), PSA (E), or hsp70-1 (F). " p<0.05: DL3 vs. CTR. Data shown were one representative experiment of three.

which occurred much earlier and peaked at $\sim 8 \mathrm{~h}$ after an incubation in fresh medium, followed by a continuous decline to approximately basal level at $48 \mathrm{~h}$ (Fig. 2E and F). Treatment with $20 \mu \mathrm{M}$ of DL3 reduced respective mRNA expression of all three genes (Fig. 2D-F). However, a significant reduction of AR mRNA occurred earlier at $\sim 4 \mathrm{~h}$ and those of PSA and hsp70-1 mRNA at $8 \mathrm{~h}$ after an exposure to DL3. These data concluded that DL3 downregulated AR, PSA, and hsp70-1 at both protein and mRNA levels and its effects were more significant at mRNA levels.

AR-dependent downregulation of hsp70-1 by DL3. Next, effects of DL3 on hsp70-1 expression were further studied in several other human prostatic cell lines with different properties, including LAPC-4 (cancer cells with wild-type AR), 22Rv1 (cancer cells with mutant AR), two AR negative cancer cell lines (PC-3, DU-145), and two transformed AR-negative prostatic epithelial cell lines (WPE1-NB14 and WPE1-NB26). LAPC-4 cells were routinely maintained in medium supplemented with $10 \mathrm{nM}$ of DHT. To be consistent with DHT concentration used in other experiments in this study, $1 \mathrm{nM}$ of DHT was used in the experiment and appeared to cause a moderate reduction of hsp70 in the cells (Fig. 3A). DL3 downregulated hsp70-1 expression in LAPC-4 cells, which appeared to be more significant in cells treated with DHT
(Fig. 3A). 22Rv1 cells were relatively refractory to androgen and expressed a relatively lower level of AR and PSA in comparison with those in LNCaP cells (data not shown). A significant downregulation of hsp70-1 in 22Rv1 cells required higher concentration (20-40 $\mu \mathrm{M})$ of DL3 (Fig. 3B). The treatment with DL3 at concentrations up to $40 \mu \mathrm{M}$ did not significantly alter hsp70-1 expression in all the AR-negative cell lines (PC-3, DU-145, PWE-NB14, and PWE-NB26 cells) (Fig. 3C). The hsp70-1 protein expression was further investigated in PC-3 cells stably engineered with recombinant AR. As shown in Fig. 3D, treatment of DL3 did not alter hsp70-1 level in parental PC-3 cells but it did reduce hsp70-1 protein expression in cells engineered with AR (Fig. 3D). On the other hand, whereas knocking down AR expression in LNCaP cells by using AR-specific siRNA but not a scrambled control siRNA only moderately reduced hsp70-1 expression, it attenuated the inhibitory effects of DL3 (Fig. 3E). Overexpression of hsp70-1 enhanced AR expression (Fig. 3F). This hsp70-1-enhanced AR expression was also reduced when cells were treated with DL3 (Fig. 3F). These data suggested that downregulation of hsp70-1 expression by DL3 in prostate cancer cells relied on the presence of AR and downregulation of hsp70-1 in human prostate cancer cells by DL3 was due possibly to an attenuation of AR expression or AR-mediated signaling. 
A

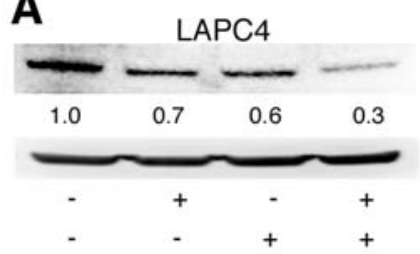

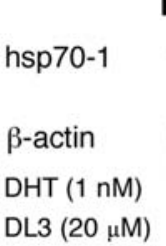

B

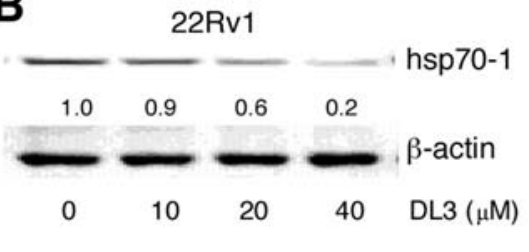

C

PC-3

DU-145

PWE-NB14

PWE-NB26

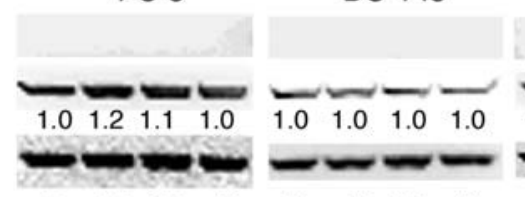

$0 \quad 10 \quad 20 \quad 40$

$\begin{array}{llll}0 & 10 & 20 & 40\end{array}$

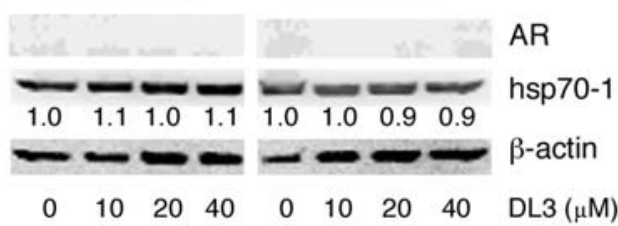

$D_{\mathrm{PC}-3}$ PC-3-AR

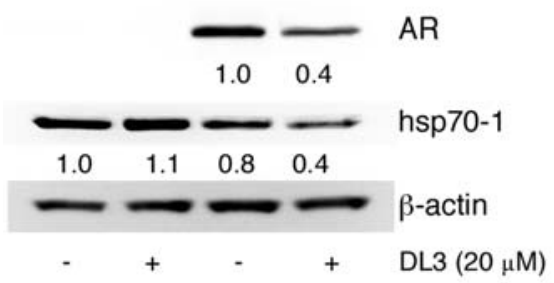

E
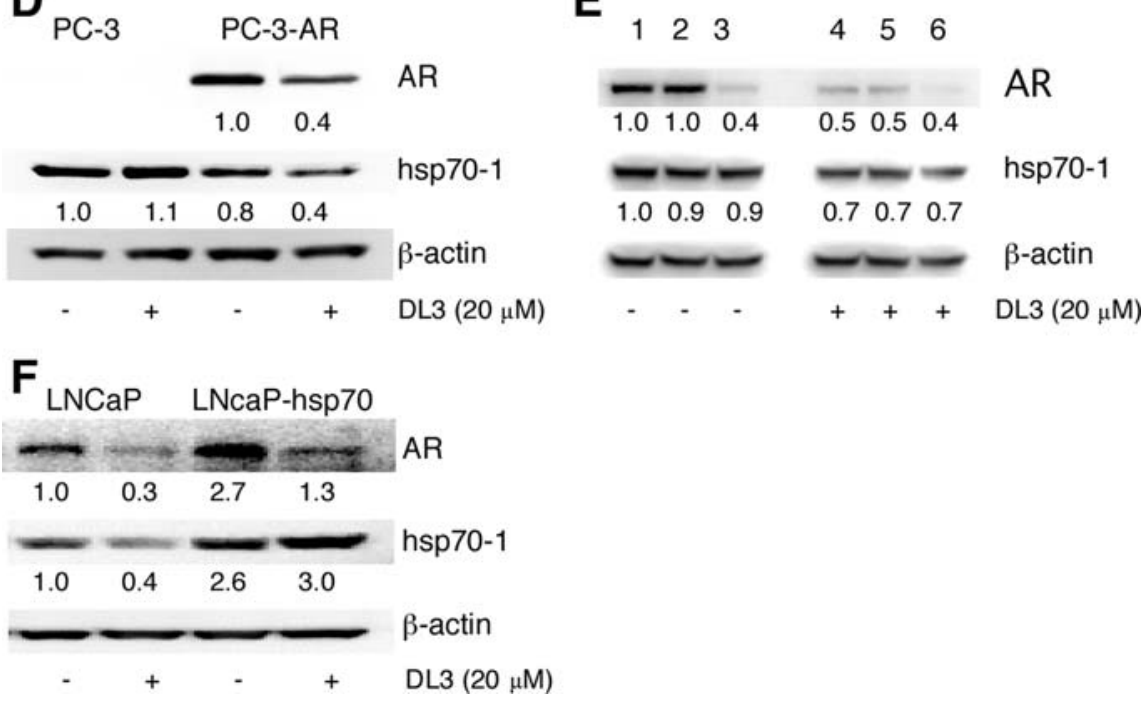

Figure 3. AR-dependent downregulation of hsp70-1 by DL3. (A) LAPC-4 cells were incubated for $48 \mathrm{~h}$ in IMDM medium supplemented with $10 \%$ SFBS and then treated for $24 \mathrm{~h}$ with $1 \mathrm{nM}$ of DHT and/or $20 \mu \mathrm{M}$ of DL3. (B) $22 \mathrm{Rv} 1$ cells $\left(2 \times 10^{6}\right.$ cells/well in a 6-well plate) in medium supplemented with $10 \%$ FBS were treated for $24 \mathrm{~h}$ with different concentrations of DL3. (C) AR-negative PC-3, DU-145, PWE-NB14, and PWE-NB26 cells were treated for 24 h with different concentrations of DL3. (D) PC-3 or PC-3AR, which were stably transfected with wild-type AR were treated for 24 h with $20 \mu \mathrm{M}$ of DL3. (E) LNCaP cells were transfected with AR-specific siRNA (lanes 3 and 6) or a scrambled siRNA (lanes 2 and 5) for $48 \mathrm{~h}$ and treated with $20 \mu \mathrm{M}$ DL3 (lanes 4-6) for $24 \mathrm{~h}$. Expression of AR and hsp70-1 in control and treated cells was analyzed by immunoblotting. (F) LNCaP cells were transfected with vector or vector expressing hsp70-1 for $24 \mathrm{~h}$ and treated for $24 \mathrm{~h}$ with DL3. Lysates from cells described above were analyzed by immunoblotting.

Effects of DL3 on AR and hsp70-1 stability and gene transcription. The degradation of both AR and hsp70-1 was shown to be mediated by the ubiquitin-mediated proteosome pathway. We, therefore, investigated whether DL3 accelerated the degradation of these two proteins in proteosome. LNCaP cells were treated with DL3 $(20 \mu \mathrm{M})$ and/or a proteosome inhibitor MG132 (5 $\mu \mathrm{g} / \mathrm{ml})$ for $12 \mathrm{~h}$. As shown in Fig. 4A, AR expression was downregulated in cells treated with DL3 (lane 1 vs. lane 2). MG132 treatment alone did not significantly alter AR expression (lane 3 vs. lane 1). It, however, abrogated AR downregulation by DL3 (lane 4 vs. lane 2), suggesting that DL3-induced AR downregulation was due to an acceleration of the proteosome-mediated degradation of AR. Hsp70-1 protein level was significantly elevated in cells treated with MG132 (lane 3 vs. lane 1). In contrast to AR expression, hsp70-1 protein level was significantly reduced by DL3 regardless of the absence (lane 2 vs. lane 1) or presence (lane 4 vs. lane 3 ) of MG132. Consistent with the observations in previous experiments, expression of hsp90 was not altered in cells treated with DL3 and/or MG132. These data suggest that
DL3 downregulated AR and hsp70-1 by different mechanisms and that it downregulated hsp70-1 expression via mechanisms independent of proteosome-mediated degradation.

Effects of DL3 on AR and hsp70-1 protein stability were further studied in cells treated with protein synthesis inhibitor cycloheximide (CHX). In androgen-starved LNCaP cells, treatment with DL3 further reduced AR level by $\sim 50 \%$ (Fig. 4B, lane 2 vs. lane 1). AR level was elevated by the presence of DHT (Fig. 4B, lane 3 vs. lane 1), which was abrogated by DL3 (Fig. 4B, lane 4 vs. lane 3). AR expression was diminished in cells treated with $\mathrm{CHX}$, which was reversed by DHT (Fig. 4B, lane 6 vs. lane 5), due to the enhancement of AR stability by DHT $(28,29)$. This effect of DHT on AR expression was abrogated by DL3 (Fig. 4B, lane 7), suggesting that DL3 promoted AR degradation. In contrast, treatment with DL3 downregulated hsp70-1 by $\sim 40 \%$ regardless of the presence or absence of either CHX and/or DHT, suggesting that DL3 did not alter hsp70-1 degradation.

Since hsp70-1 mRNA level was significantly reduced in cells treated with DL3, we determined whether DL3 regulated 


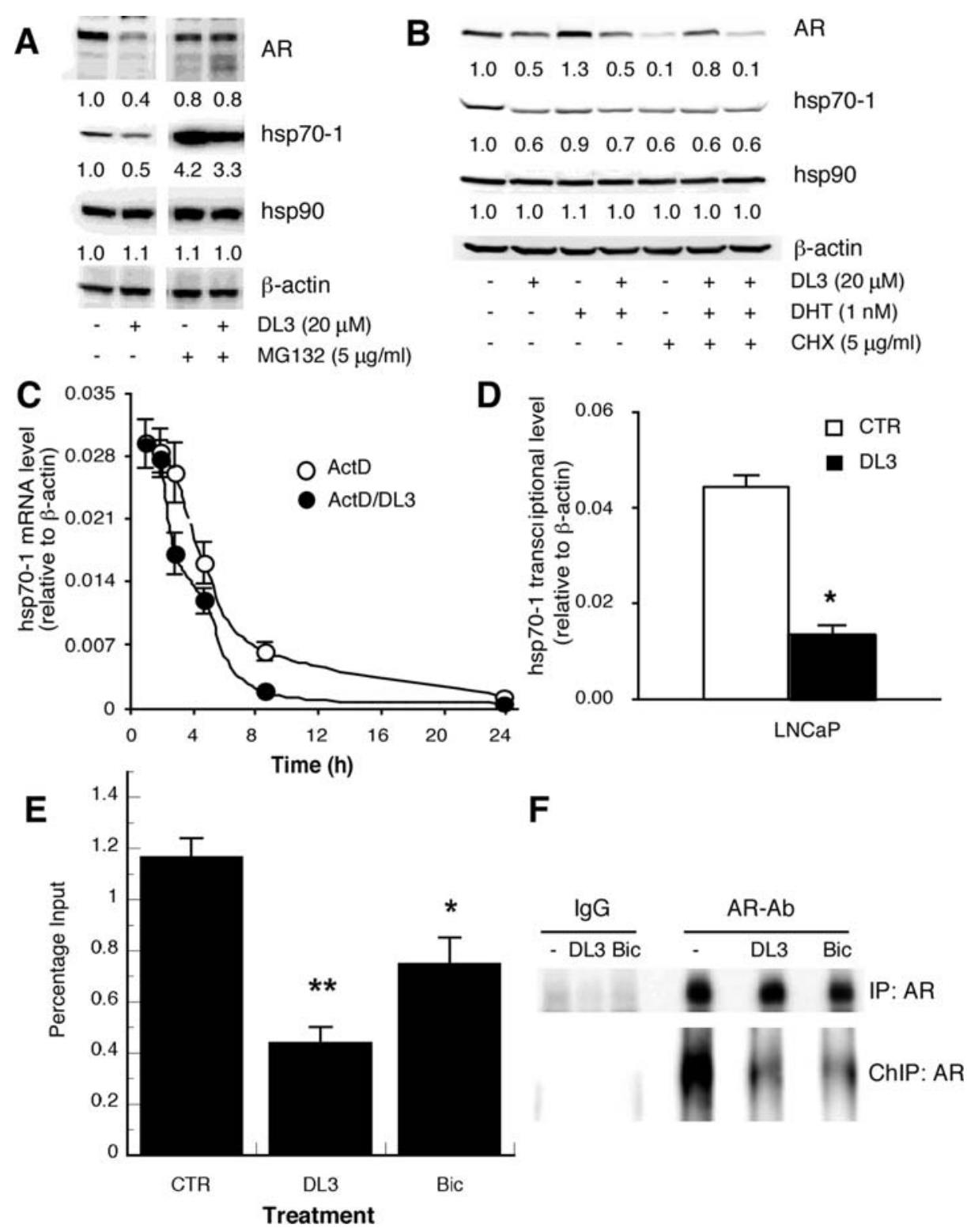

Figure 4. Mechanistic study on DL3-induced hsp70-1 downregulation. (A) LNCaP cells in medium supplemented with $10 \%$ FBS were treated for $16 \mathrm{~h}$ with $20 \mu \mathrm{M}$ of DL3 and/or $5 \mu \mathrm{g} / \mathrm{ml}$ of MG132. (B) LNCaP cells in medium supplemented with 10\% SFBS were treated for $24 \mathrm{~h}$ with $20 \mu \mathrm{M}$ of DL3, $1 \mathrm{nM}$ of DHT, and $/$ or $5 \mu \mathrm{g} / \mathrm{ml}$ of CHX. (C) LNCaP cells were treated for different times with $5 \mu \mathrm{g} / \mathrm{ml}$ of ActD in the absence or presence of $20 \mu \mathrm{M}$ of DL3. Total RNA was extracted and analyzed by qRT-PCR. (D) LNCaP cells, incubated in medium or treated with $20 \mu \mathrm{M}$ of DL3, were lyzed and mixed with the same volume of $0.6 \mathrm{M}$ isolation sucrose buffer. Cell nucleus pellets obtained were incubated in transcription reaction buffer. Total RNA in the reaction mixture was extracted, and hsp70-1 and B-actin mRNA was assessed by real-time RT-PCR. Samples without in vitro transcription reaction were used as controls (not shown). (E) LNCaP cells, treated for $4 \mathrm{~h}$ with $20 \mu \mathrm{M}$ of DL3 or Bic, were fixed in $1 \%$ formaldehyde. After washing and incubation in glycine, cells were scrapped into PBS supplemented with $5 \mathrm{mM}$ PMSF, pelleted, resuspended in SDS lysis buffer, and sonicated to sheer DNA. AR-chromatintranscription factor complex was precipitated with antibodies to AR with IgG from normal rabbit as a negative control. With 1:150 dilution of total DNA as a positive control (total input), DNA obtained from the immunoprecipitation was analyzed by using qPCR. (F) A portion of the ChIP samples was analyzed by immunoblotting to evaluate AR levels. In a parallel setting, total cell lysates were precipitated with the same AR antibody to evaluate the total cellular AR level in each treatment group.

hsp70-1 mRNA stability. Cells were treated with $5 \mu \mathrm{g} / \mathrm{ml}$ of actinomycin D (ActD) to block de novo synthesis of new RNA and with DL3 $(20 \mu \mathrm{M})$ for various times. Data in Fig. 4C show that hsp70-1 had a short half-life of about $4 \mathrm{~h}$. The half-life of hsp70-1 mRNA was not significantly altered in cells treated with DL3 (Fig. 4C). On the other hand, the nuclear run-on assay showed that transcription of hsp70-1 mRNA in vitro by nuclear extracts from DL3-treated cells was reduced $\sim 60-70 \%$ (Fig. 4D), suggesting downregulation of hsp70-1 mRNA and, hence, hsp70-1 protein by DL3 was due mainly to suppression of hsp70-1 gene transcription.
To further explore the potential role of AR in regulating hsp70-1 expression, we first performed sequence analysis of both HSPA1A and HSPA1B gene promoters and identified two consensus sequences of androgen response element (ARE) for each of the genes in the core promoter region spanning $\sim 450$ bp including both 5'UTR and 250 bp core promoter. ChIP assay was performed to determine whether AR occupied $H S P A-1 B$ gene promoter region. LNCaP cells were incubated for $4 \mathrm{~h}$ in medium supplemented with $10 \%$ FBS without or with treatment of $20 \mu \mathrm{M}$ of DL3 or Bic. Subsequently, the cells were fixed. Sheared chromatin was immunoprecipitated 
Cell membrane

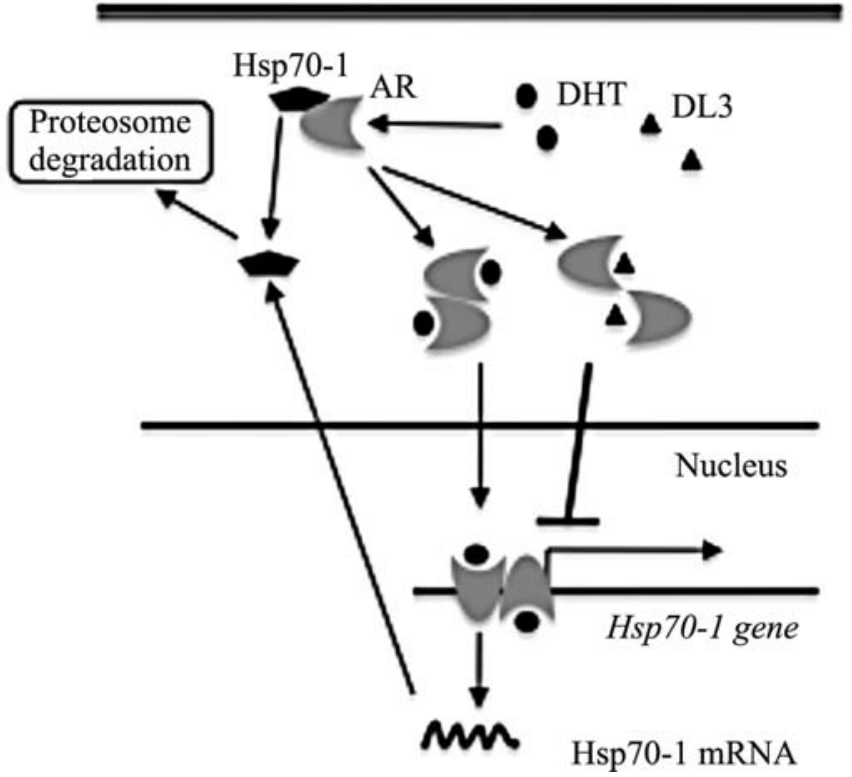

Figure 5. Putative mechanisms regulating hsp70-1 expression by AR and AR signaling antagonists.

with control rabbit IgG (data not shown) or AR-specific antibody. The chromatin precipitate was amplified using a primer pair (27) corresponding to the promoter region of $H S P A 1 B$ gene with total DNA (1:150 dilution) as positive control (total input). As shown in Fig. 4E, AR bound to HSPAIB promoter region which was reduced by approximately 60 and $35 \%$ in cells treated with DL3 and Bic, respectively. The immunoblot analysis showed that treatment for $4 \mathrm{~h}$ with DL3 or Bic did not significantly alter the total cellular AR levels, but reduced the chromatin-associated AR in the ChIP samples (Fig. 4F), suggesting that the treatments reduced the occupation of AR to its target genes.

\section{Discussion}

The hsp70-1 proteins are molecular chaperons critical for AR biosynthesis and function (1,6-8). Its expression and regulation in prostate cancer cells are, however, not well studied. Data presented in this report show that hsp70-1 is constitutively expressed in all human prostatic epithelial cell lines examined, including AR-positive androgen-dependent LNCaP and LAPC-4 prostate cancer cells, AR-positive androgen-independent $22 \mathrm{Rv} 1$ prostate cancer cells, ARnegative androgen-independent PC-3 and DU145 prostate cancer cells, and transformed prostatic epithelial WPE1-NB14 and WPE1-NB26 cells. Treatment of androgen-dependent LNCaP cells with DHT did not significantly alter hsp70-1 protein level but enhances hsp70-1 gene transcription and mRNA synthesis. Expression of hsp70-1, but not hsp90, is downregulated by AR signaling antagonist DL3 and by antiandrogens Bic and Flut. The downregulation of hsp70-1 expression by DL3 requires the presence of $\mathrm{AR}$ in the target cells, regardless of their androgen-dependency status, and is observed in PC-3 cells forced to express AR. These findings strongly suggest that $\mathrm{AR}$ and its signaling regulate the expression level of hsp70-1 expression in human prostate cancer cells.

It appears that $\mathrm{AR}$ and, hence, $\mathrm{AR}$ antagonists regulate hsp70-1 expression in prostate cancer cells mainly at the transcription level. This conclusion is supported by several lines of evidence. First, DHT does not significantly alter hsp70-1 protein level but enhances hsp70-1 mRNA expression, which is blocked by DL3. Second, the blockades of protein synthesis using $\mathrm{CHX}$ and protein degradation using MG132 do not reverse or attenuate the inhibitory effects of DL3 on hsp70-1 expression. Third, DL3 treatment does not significantly accelerate hsp70-1 mRNA degradation. Forth, DL3 significantly reduces the transcription of hsp70-1 gene in vitro in the nuclear run-on assay. Finally, AR occupies the promoter region of hsp70-1 gene, which is reduced upon a treatment with DL3 or Bic, and the reduction levels correlate with potencies of the inhibitory effects of DL3 and Bic on hsp70-1 mRNA and protein expression. Together with the fact that hsp70-1 is associated with AR in the cytoplasm and undergoes proteosome-mediated degradation upon dissociation from AR in cells exposing to androgen, findings in this study implicate that AR activation induces hsp70-1 expression. This is possibly a feedback mechanism that maintains a relative constant level of hsp70-1 prostate cancer cells (Fig. 5). In AR-positive cells treated with DL3 or an antiandrogen, however, the AR-induced hsp70-1 expression is attenuated and, hence, leads to a downregulation of hsp70-1. On the other hand, the lack of AR in several AR-negative cell lines renders their refractory to DL3-mediated downregulation of hsp70-1, which is reversed when cells are forced to express AR. Consistent with this notion, the knocking down of AR leads a moderate reduction of hsp70-1 level in the cells and abolishes the downregulation of hsp70-1 by DL3.

Several earlier studies showed that hsp70-1 expression levels vary with the cell cycle and are regulated by c-Myc (30-33). The hsp70-1 expression level is elevated by $65 \%$ in $\mathrm{G} 2 / \mathrm{M}$ phase of the cell cycle and is lowest in G1 phase among G1, S, and G2/M2 phases of the cell cycle (34). Cultivation of LNCaP cells under androgen-deprived conditions arrests cells at G1 phase of the cell cycle (35). Similarly, treatment with the classical antiandrogens Bic and Flut, and DL3 induce G1 arrest in LNCaP cells (data not shown). Therefore, it is conceivable that reduction of hsp70-1 expression in cells treated by DL3 and the classical antiandrogens is due indirectly to the alteration of cell cycle progression. The observations that reduction of hsp70-1 mRNA, simultaneously with PSA, occurs very early (in $8 \mathrm{~h}$ ) after treatment with DL3 and that AR binds to HSPA1B gene promoter region, however, argue against this notion. Taken together, whereas further studies are needed to establish and elucidate mechanisms by which AR regulates hsp70-1 expression in prostatic cells, data presented in this report do strongly suggest that AR and/or AR signaling may directly regulate hsp70-1 gene transcription.

Previous studies have shown that the bioflavonoid quercetin is a potent inhibitor of hsp70-1 expression. Quercetin downregulates both constitutive and heat-induced synthesis of hsp70-1, inhibits cell growth, and promotes heat-induced apoptosis in a variety of cell lines, including prostate cancer cells (36-40). Interestingly, quercetin also inhibits AR expression and androgen-induced expression of PSA and 
other AR target genes (41-43), which is similar to that observed by us with DL3 (20). It is, therefore, of interest to further compare effects of DL3 and quercetin on hsp70-1 expression in both prostate cancer and other types of cells. In addition, it is also of interest in determining whether DL3 could regulate heat-induced hsp70-1 expression in both AR-negative and AR-positive cells. Not only do these studies facilitate the elucidation of roles of AR signaling in regulating hsp70-1 expression, but they may also demonstrate whether DL3 can serve as a potential heat-sensitizer agent for thermal therapy against prostate cancer.

In conclusion, data presented in this study show that: i) DHT enhances hsp70-1 mRNA expression in human prostate cancer cells, which is attenuated by DL3; ii) DL3, as well as antiandrogens Bic and Flut, downregulates hsp70-1 expression in an AR-dependent fashion and occurs at transcription level; and iii) AR occupies the promoter region of $H S P A 1 B$ gene, which is reduced by DL3 and Bic. These data conclude that AR and its signaling regulate hsp70-1 expression in prostate cancer cells. Given the finding that knockdown expression of hsp70-1 sensitizes prostate cancer cells to chemotherapies as well as other therapeutic modalities (9), downregulation of hsp70-1 using AR signaling antagonist DL3 or an antiandrogen may present an attractive strategy for successful management of advanced prostate cancer through combinational therapies.

\section{Acknowledgements}

This work is supported in part University of Cincinnati Cancer Center and NIH-NCI R01 CA131137A1. The authors would like to thank Dr Zhengxin Wang for providing AR-engineered PC-3AR cells, Dr Robert Reiter for the approval to use LAPC-4 cells in our laboratory, Dr Karen Knudsen for providing LAPC-4.

\section{References}

1. Calderwood SK, Khaleque MA, Sawyer DB and Ciocca DR: Heat shock proteins in cancer: chaperones of tumorigenesis. Trends Biochem Sci 31: 164-172, 2001.

2. Lindquist $\mathrm{S}$ and Craig EA: The heat-shock proteins. Annu Rev Genet 22: 631-677, 1988.

3. Daugaard M, Rohde M and Jaattela M: The heat shock protein 70 family: highly homologous proteins with overlapping and distinct functions. FEBS Lett 581: 3702-3710, 2007.

4. Mayer MP and Bukau B: Hsp70 chaperones: cellular functions and molecular mechanism. Cell Mol Life Sci 62: 670-684, 2005

5. Brodsky JL and Chiosis G: Hsp70 molecular chaperones: emerging roles in human disease and identification of small molecule modulators. Curr Top Med Chem 6: 1215-1225, 2006.

6. Veldscholte J, Berrevoets CA, Brinkmann AO, Grootegoed JA and Mulder E: Anti-androgens and the mutated androgen receptor of LNCaP cells: differential effects on binding affinity, heat-shock protein interaction, and transcription activation. Biochemistry 31: 2393-2399, 1992.

7. Bohen SP, Kralli A and Yamamoto KR: Hold 'em and fold 'em: chaperones and signal transduction. Science 268: 1303-1304, 1995.

8. Shatkina L, Mink S, Rogatsch H, et al: The cochaperone Bag-1L enhances androgen receptor action via interaction with the NH2terminal region of the receptor. Mol Cell Biol 23: 7189-7197, 2003.

9. Gabai VL, Budagova KR and Sherman MY: Increased expression of the major heat shock protein Hsp72 in human prostate carcinoma cells is dispensable for their viability but confers resistance to a variety of anticancer agents. Oncogene 24 : 3328-3338, 2005.
10. Barnes JA, Dix DJ, Collins BW, Luft C and Allen JW: Expression of inducible Hsp70 enhances the proliferation of MCF-7 breast cancer cells and protects against the cytotoxic effects of hyperthermia. Cell Stress Chaperones 6: 316-325, 2001.

11. Garrido C, Brunet M, Didelot C, Zermati Y, Schmitt E and Kroemer G: Heat shock proteins 27 and 70: anti-apoptotic proteins with tumorigenic properties. Cell Cycle 5: 2592-2601, 2006.

12. Beere HM and Green DR: Stress management - heat shock protein-70 and the regulation of apoptosis. Trends Cell Biol 11: 6-10, 2001.

13. Ren A, Yan G, You B and Sun J: Down-regulation of mammalian sterile 20 -like kinase 1 by heat shock protein 70 mediates cisplatin resistance in prostate cancer cells. Cancer Res 68: 2266-2674, 2008

14. Powers MV, Clarke PA and Workman P: Dual targeting of HSC70 and HSP72 inhibits HSP90 function and induces tumorspecific apoptosis. Cancer Cell 14: 250-262, 2008.

15. Schmitt E, Parcellier A, Gurbuxani S, et al: Chemosensitization by a non-apoptogenic heat shock protein 70-binding apoptosisinducing factor mutant. Cancer Res 63: 8233-8240, 2003.

16. Garrido C, Schmitt E, Cande C, Vahsen N, Parcellier A and Kroemer G: HSP27 and HSP70: potentially oncogenic apoptosis inhibitors. Cell Cycle 2: 579-584, 2003.

17. Ravagnan L, Gurbuxani S, Susin SA, et al: Heat-shock protein 70 antagonizes apoptosis-inducing factor. Nat Cell Biol 3: 839-843, 2001.

18. Liu J, Tsang S and Wong TM: Testosterone is required for delayed cardioprotection and enhanced heat shock protein 70 expression induced by preconditioning. Endocrinology 147: 4569-4577, 2006

19. Xiao N and De Franco DB: Overexpression of unliganded steroid receptors activates endogenous heat shock factor. Mol Endocrinol 11: 1365-1374, 1997.

20. Lu S, Wang A, Lu S and Dong Z: A novel synthetic compound that interrupts androgen receptor signaling in human prostate cancer cells. Mol Cancer Ther 6: 2057-2064, 2007.

21. Horoszewicz JS, Leong SS, Kawinski E, et al: LNCaP model of human prostatic carcinoma. Cancer Res 43: 1809-1818, 1983.

22. Van Bokhoven A, Varella-Garcia M, Korch C, et al: Molecular characterization of human prostate carcinoma cell lines. Prostate 57: 205-225, 2003.

23. Sramkoski RM, Pretlow TG II, Giaconia JM, et al: A new human prostate carcinoma cell line, 22Rv1. In Vitro Cell Dev Biol Anim 35: 403-409, 1999.

24. Klein KA, Reiter RE, Redula J, et al: Progression of metastatic human prostate cancer to androgen independence in immunodeficient SCID mice. Nat Med 3: 402-408, 1997.

25. Dong Z, Liu Y, Lu S, et al: Vav3 oncogene is overexpressed and regulates cell growth and androgen receptor activity in human prostate cancer. Mol Endocrinol 20: 2315-2325, 2006.

26. Lu S and Dong Z: Characterization of TGF-beta-regulated interleukin-8 expression in human prostate cancer cells. Prostate 66: 996-1004, 2006.

27. Han Q, Hou X, Su D, et al: hELP3 subunit of the elongator complex regulates the transcription of HSP70 gene in human cells. Acta Biochim Biophys Sin 39: 453-461, 2007.

28. Yeap BB, Krueger RG and Leedman PJ: Differential posttranscriptional regulation of androgen receptor gene expression by androgen in prostate and breast cancer cells. Endocrinology 140: 3282-3291, 1999.

29. Zhou ZX, Lane MV, Kemppainen JA, French FS and Wilson EM: Specificity of ligand-dependent androgen receptor stabilization: receptor domain interactions influence ligand dissociation and receptor stability. Mol Endocrinol 9: 208-218, 1995.

30. Taira T, Sawai M, Ikeda M, Tamai K, Iguchi-Ariga SM and Ariga H: Cell cycle-dependent switch of up-and down-regulation of human hsp70 gene expression by interaction between c-Myc and CBF/NF-Y. J Biol Chem 274: 24270-24279, 1999.

31. Moreau N, Prudhomme C and Angelier N: Cell-cycle-dependent nuclear translocation of HSP70 in amphibian embryonic cells. Int J Dev Biol 42: 633-636, 1998.

32. Milarski KL and Morimoto RI: Expression of human HSP70 during the synthetic phase of the cell cycle. Proc Natl Acad Sci USA 83: 9517-9521, 1986.

33. Wu BJ and Morimoto RI: Transcription of the human hsp70 gene is induced by serum stimulation. Proc Natl Acad Sci USA 82: 6070-6074, 1985 . 
34. Hang $\mathrm{H}$, He L and Fox MH: Cell cycle variation of Hsp70 levels in HeLa cells at 37 degrees $\mathrm{C}$ and after a heat shock. $\mathrm{J}$ Cell Physiol 165: 367-375, 1995.

35. Knudsen K, Fribourg AF, Petre C and Wetherill Y: Androgen mediated regulation of the G1-S transition in prostate cancer In: Steroid Hormones and Cell Cycle Regulation. Burnstein KL (ed). Kluwer Academic Publishers, Boston, MA, pp1-110, 2002.

36. Ma ZS, Huynh TH, Ng CP, Do PT, Nguyen TH and Huynh H: Reduction of CWR22 prostate tumor xenograft growth by combined tamoxifen-quercetin treatment is associated with inhibition of angiogenesis and cellular proliferation. Int J Oncol 24: 1297-1304, 2004.

37. Kobayashi T, Nakata $\mathrm{T}$ and Kuzumaki T: Effect of flavonoids on cell cycle progression in prostate cancer cells. Cancer Lett 176: 17-23, 2002.

38. Wei YQ, Zhao X, Kariya Y, Fukata H, Teshigawara K and Uchida A: Induction of apoptosis by quercetin: involvement of heat shock protein. Cancer Res 54: 4952-4957, 1994.
39. Hansen RK, Oesterreich S, Lemieux P, Sarge KD and Fuqua SA: Quercetin inhibits heat shock protein induction but not heat shock factor DNA-binding in human breast carcinoma cells. Biochem Biophys Res Commun 239: 851-856, 1997.

40. Nakanoma T, Ueno M, Iida M, Hirata R and Deguchi N: Effects of quercetin on the heat-induced cytotoxicity of prostate cancer cells. Int J Urol 8: 623-630, 2001

41. Yuan H, Pan Y and Young CY: Overexpression of c-Jun induced by quercetin and resverol inhibits the expression and function of the androgen receptor in human prostate cancer cells. Cancer Lett 213: 155-163, 2004.

42. Xing N, Chen Y, Mitchell SH and Young CY: Quercetin inhibits the expression and function of the androgen receptor in $\mathrm{LNCaP}$ prostate cancer cells. Carcinogenesis 22: 409-414, 2001.

43. Culig Z, Klocker H, Bartsch G and Hobisch A: Androgen receptors in prostate cancer. Endocr Relat Cancer 9: 155-170, 2002. 